\title{
Etkin Yük Yaklaşımı Kullanarak Protonlar için Z=2-54 Elementlerin Durdurma Gücü Üzerine Bir Çalışma
}

\author{
Metin Usta ${ }^{1 *}$ \\ ${ }^{1}$ Mustafa Kemal Üniversitesi, Fen Edebiyat Fakültesi, Fizik Bölümü, 31034, Hatay
}

Geliş / Received: 08.11.2018, Kabul / Accepted: 15.12.2019

\section{Öz}

Durdurma gücü, hedef içerisindeki elektronlar ve çekirdeğin Coulomb alanı etkileşmesi nedeniyle yüklü parçacıkların birim uzunluk başına ortalama enerji kaybıdır. Durdurma gücünün atom fiziği, nükleer fizik ve uygulamaları, radyasyon dozimetrisi gibi birçok alanlarda önemli uygulamaları vardır. Bu çalışmanın amacı 1 keV-1 GeV enerjili protonlar için $\mathrm{Z}=2-54$ atom numaralı elementlerin Bethe-Bloch teorisine dayalı etkin yük yaklaşımı yöntemi kullanılarak çarpışma durdurma gücünü hesaplamaktır. Bunun için öncelikle hedef elementlerin etkin yük, etkin ortalama uyarılma enerjileri ve potansiyel enerji fonksiyonları belirlendi. Elektronik yük yoğunluğu için Roothaan-Hartree-Fock atomik dalgafonksiyonları seçildi ve hedef atomların etkin yükünün protonların enerjisiyle değişimi incelendi. Ardından elde edilen hedef atomların etkin yükleri kullanılarak çarpışma durdurma gücü değerleri hesaplandı. Atom numarası ve gelen protonların enerjisi arttıkça etkin yük değerlerinin arttığg görüldü. Durdurma gücü değerlerinin ise atom sayısı arttıkça azaldığ 1 ve durdurma gücüne ait maksimum noktalarının yüksek enerjilere doğru kaydığı belirlendi. Hedef elementlerden karbon atomu için hesaplanan durdurma gücü değerleri literatürde mevcut ICRU 49, SRIM, CasP ve Janni'nin verileriyle karşılaştırıldı. Sonuçların belirli hata oranlarında literatür verileriyle oldukça uygun oldukları gözlendi. Ayrıca bu çalışmada kullanılan etkin yük yaklaşımının $1 \mathrm{MeV}$ 'den düşük enerjilerde de kullanılabileceği anlaşıldı.

Anahtar Kelimeler: Durdurma gücü, Etkin yük, Bethe-Bloch, Proton

\section{A Study on The Stopping Power of $Z=2-54$ Elements for Protons Using Effective-Charge Approximation}

\begin{abstract}
The stopping power is the average energy loss per unit length of charged particles due to the interaction with the Coulomb field of electrons in target and nucleus. Stopping power has important applications in many areas such as atomic physics, nuclear physics and applications, radiation dosimetry. The aim of this study is to calculate the collision stopping power of $\mathrm{Z}=2-54$ atomic elements by using the effective charge approach based on Bethe-Bloch theory for the $1 \mathrm{keV}-1 \mathrm{GeV}$ energy protons. For this purpose, the effective charge, effective mean excitation energies and potential energy functions of the target elements were firstly determined. For the electronic charge density, Roothaan-Hartree-Fock atomic wavefunctions were selected and the change of the effective charge of the target atoms with the energy of the protons was examined. Then, the collision stopping power values were calculated by using the obtained effective charges of the target atoms. It was seen that as the atomic number and the energy of the incoming protons increased, effective charge values ascended. It was determined that the stopping power values decreased as the number of atoms increased and the maximum points of stopping power shifted towards high energies. The calculated stopping power values for the carbon atom from the target elements were compared with the data of the ICRU 49, SRIM, CasP and Janni available in the literature. It was observed that the results were quite appropriate with the literature data at certain error rates. In addition, it is understood that the effective charge approach used in this study can be used in energies less than $1 \mathrm{MeV}$.
\end{abstract}

Keywords: Stopping power, Effective charge, Bethe-Bloch, Proton 


\section{Giriş}

Durdurma gücü, hedef içerisindeki elektronlar ve çekirdeğin Coulomb alanı ile etkileşmesi nedeniyle yüklü parçacıkların birim uzunluk başına ortalama enerji kaybıdır. Durdurma gücünün atom fiziği, nükleer fizik ve radyasyon dozimetrisi gibi birçok alanlarda önemli uygulamaları vardır (Dib et al., 2015). Farklı hedef ve iyonlar için durdurma gücü ile ilgili birçok teorik ve deneysel çalışmalar mevcuttur (Damache, Ouichaoui, Belhout, Medouni, \& Toumert, 2004; Demir et al., 2015; Garcia-Molina, Abril, de Vera, \& Paul, 2013; Özdoğan, Çapalı, \& Kaplan, 2015; Pierce, Bowman, \& Blann, 1968; Porter, 1980; Reynolds, Dunbar, Wenzel, \& Whaling, 1953; Sarpün et al., 2015; J. F. Ziegler \& Manoyan, 1988). Bununla birlikte, protonlar için periyodik tablodaki çoğu elementlere ait sınırlı sayıda tablolanmış durdurma gücü değerleri ve bu hesaplamaya yönelik programlar vardir (Agostinelli et al., 2003; Ferrari, 2005; Grande \& Schiwietz, 2009; ICRU, 1993; Janni, 1982; J.F. Ziegler, Biersack, \& Ziegler, 2013). Dolayısıyla periyodik tablodaki hedef elementlere ait etkin yük ve durdurma gücünün gelen protonların enerjisine göre değişimini belirlemeye ihtiyaç duyulmaktadır.

$\mathrm{Bu}$ çalışmanın amacı $1 \mathrm{keV}-1 \mathrm{GeV}$ enerjili protonlar için $\mathrm{Z}=2-54$ atom numaralı elementlerin Bethe-Bloch teorisine dayalı etkin yük yaklaşımı yöntemi kullanılarak çarpışma durdurma gücünü hesaplamaktır. Bunun için öncelikle hedef elementlerin etkin yük, etkin ortalama uyarılma enerjileri ve potansiyel enerji fonksiyonları belirlendi. Elektronik yük yoğunluğu için RoothaanHartree-Fock (RHF) atomik dalgafonksiyonları seçildi ve hedef atomların etkin yükünün protonların enerjisiyle değişimi incelendi. Ardından elde edilen hedef atomların etkin yükleri kullanılarak çarpışma durdurma gücü değerleri hesaplandı. Hedef elementlerden karbon atomu için hesaplanan durdurma gücü değerleri literatürde mevcut ICRU 49 (ICRU, 1993), SRIM (J.F. Ziegler et al., 2013), CasP (Grande \& Schiwietz, 2009) ve Janni'nin (Janni, 1982) verileriyle karşılaştırıldı. Sonuçların belirli hata oranlarında literatür verileriyle oldukça uyumlu oldukları gözlendi. Ayrıca bu çalışmayla etkin yük yaklaşımının $1 \mathrm{MeV}$ 'den düşük enerjilerde de kullanılabileceği anlaşıldı.

\section{Materyal ve Metot}

\section{1. Çarpışma durdurma gücü}

$M$ kütleli ve $Z e$ yüklü durgun bir hedef atom üzerine gelen $v$ hızına sahip bir proton düşünelim. Hedef atom ve proton arasındaki etkileşme potansiyeli aşağıdaki gibi yazılabilir:

$$
\mathrm{V}(x, y, R)=\frac{Z e^{2}}{R}-\sum_{i=1}^{Z^{*}} \frac{e^{2}}{\left|R-y_{i}\right|}
$$

$$
\langle\mathrm{V}\rangle=\frac{4 \pi e^{2}}{q^{2}}\left[Z \delta_{m m_{0}}-M_{m m_{0}}(q)\right]
$$

Burada $Z^{*}$ hedefin etkin yükü, $q$ ise momentum transferidir. Ayrıntılar için Referans 12'deki (Cabrera-Trujillo, Cruz, Oddershede, \& Sabin, 1997) çalışmaya bakılabilir. Böylece diferansiyel tesir kesiti

$$
\frac{d \sigma_{n, m}}{d \Omega}=\frac{4 e^{4} m_{p}^{2}}{h^{4} q^{4}} \frac{k}{k_{0}}\left|Z \delta_{m m_{0}}-M_{m m_{0}}\right|^{2}
$$

bağıntısı ile verilir. Eğer $d \Omega=q d q d f k k_{0}=d q / k k_{0} \quad$ katı açı tanımı kullanılırsa, durdurma tesir kesiti aşağıdaki denklemden hesaplanabilir: 
$S(E)=\frac{4 \pi e^{4} m_{p}^{2}}{h^{4}} \sum_{m} \int \frac{k}{k_{0}}\left|Z \delta_{m_{0} m}-M_{m_{0} m}(q)\right|^{2} \times$

$\left|E_{m}-E_{m_{0}}\right| \frac{d \Omega}{q^{4}}$

Burada, $M_{m_{0} m}(q)$ atomik biçim çarpanıdır ve

$$
M_{m_{0} m}(q)=\left\langle m_{0}\left|\sum_{j=1}^{Z^{*}} e^{-i q y_{j}}\right| m\right\rangle
$$

ile verilir. $\mathrm{Bu}$ çalışmada, gelen proton ve hedef atomun her ikisinin de küresel simetrik olduğu kabul edildi. Buna göre atomik biçim çarpanı aşağıdaki formülden elde edilebilir:

$M_{00}(q)=4 \pi \int \frac{\rho(r) \sin (q r)}{q r} r^{2} d r$

Böylece, protonlar için çarpışma durdurma gücü

$$
S_{\text {çarp. }}(E)=\frac{4 \pi e^{4}}{m_{e} c^{2} \beta^{2}} Z^{*} \ln \left(\frac{q_{\max }}{q_{\min }}\right)
$$

olarak elde edilir. Burada, $\beta=v / c$ protonun hızının ışığın hızına oranıdır. $q_{\text {maks }}$ ve $q_{\text {min }}$ gelen protonlardan hedefe sirasiyla maksimum ve minimum momentum transferleridir (Cabrera-Trujillo et al., 1997). Ayrıntılar için (Usta \& Tufan, 2017; Usta, Tufan, Aydın, \& Bozkurt, 2018)'da bulunan çalışmalara bakılabilir.

\subsection{Etkin yük ve etkin ortalama uyarma enerjisi}

Durdurma tesir kesitini hesaplayabilmek için hedefin etkin yükü, etkin ortalama uyarma enerjisi ve atomik biçim çarpanlarının bilinmesi gereklidir. $\mathrm{Bu}$ amaçla, bu niceliklerin hesaplanmasinda Bohr sıyrılma ölçütü (Bohr, 1940, 1941) dikkate alındı.
Buna göre, hedefin etkin yükü aşağıdaki gibi hesaplanır:

$Z_{2}^{*}(v)=\int_{r_{c}}^{\infty} 4 \pi r^{2} \rho_{2}(r) d r$

Burada, $\quad r_{c}$ Bohr sıyrılma ölçütünden bulunacak sıyrılma uzaklığı, $\rho(r)$ elektronik yük yoğunluğudur. Etkin ortalama uyarılma enerjisi ise aşağıdaki bağıntıdan belirlenebilir (Sugiyama, 1981):

$\ln I^{*}=\frac{1}{Z^{*}} \int_{r_{c}}^{\infty} \ln \left[\gamma \hbar \omega_{p}(r)\right] 4 \pi r^{2} \rho(r) d r$

Formülde, $\omega_{p}$ plazma frekansı, $\gamma$ ise $\sqrt{2}$ civarında ayarlanabilir bir parametredir.

\subsection{Elektronik yük yoğunluğu}

Çarpışma durdurma gücü hesaplamaları için gerekli olan elektronik yük yoğunluğu RHF dalgafonksiyonları (Bunge, Barrientos, \& Bunge, 1993) kullanılarak hesaplandı. Genellikle Hartree-Fock uzaysal orbitallere uygun analitik ifadeler Slater determinantlarıyla sağlanır:

$\chi_{n l m}(\mathbf{r})=N r^{n-1} e^{-\alpha r} Y_{l, m}(\theta, \phi)$

Burada, $n$ pozitif bir sabit, $\alpha$ üstel katsayı, $Y_{l, m} \quad$ küresel harmonikler ve $N$ normalizasyon sabitidir. Bu çalışmada, RHF dalgafonksiyonları radyal fonksiyonların sınırlı bir süperpozisyonu olarak genişleyen radyal atomik orbitaller olarak aşağıdaki gibi alındı:

$u(\mathbf{r})=\sum_{i=1}^{N} c_{i} \chi_{i}(\mathbf{r})$

Denklemde $c_{i}$ yörünge genişleme katsayılarıdır. 


\section{Bulgular}

Bu çalışmada, $1 \mathrm{keV}-1 \mathrm{GeV}$ enerjili protonlar için hidrojen hariç $Z=54$ 'e kadar ki elementlerin çarpışma durdurma gücü hesaplanmıştır. $\mathrm{Bu}$ amaçla öncelikle hedef elementlerin etkin atom sayıları bulunmuştur.

\subsection{Etkin yük}

Şekil 1'de hedef elementlerin etkin yüklerinin gelen protonların enerjisine göre değişimi görülmektedir. Buna göre, enerji arttıkça elementlerin etkin yükleri, kendi atom sayılarına yaklaşmaktadır. Atom sayısı arttıkça, hedeflerin etkin atom sayılarındaki değişim artan enerjilere doğru kaymaktadır. Etkin atom sayılarındaki değişim yaklaşık 1$10 \mathrm{MeV}$ enerji aralığında olmaktadır.

$v$ hizı ile hareken eden ve $v_{c}$ orbital hızlarında elektron taşıyan atomlar hedefin çekirdeği ve elektronlarıyla etkileşime maruz kalmaktadır. Bohr sıyrılma kriterine göre (Bohr, 1940), elektronların $v_{c}$ orbital hızları, gelen atomların $v$ hızından küçük olduğunda bu atomlara zayıf bir şekilde bağlanır ve bu elektronlar yüksek iyonizasyon tesir kesitine sahip olduğundan dolayı bağlı olduğu atomdan sıyrılır. Bu çalışmada protonlardan ziyade hedefin sıyrılma uzaklıkları, etkin yükü ve etkin ortalama uyarılma enerjileri dikkate alındı. Dolayısıyla Bohr kriterindeki denge yük durumuna göre hedefin elementler sıyrılma kriteri tarafından öngörülenden daha fazla elektron taşıdığı için en diştaki elektronlar $v$ orbital hızına sahip olana kadar iyonizasyon baskın olacaktır. $v \square v_{c}$ hızlarında hedefin elektronları gelen protonlar tarafindan oluşturulan Coulomb etkileşmesi nedeniyle elektron yakalama veya polarizasyon bulutunun oluşumuyla enerji kaybı olayı meydana gelir. Böylece hedefin etkin yükü ortamın özelliklerinden ziyade sadece gelen protonların hizına ve hedef malzemelerin atom sayılarına bağlıdır. Dolayısıyla Şekil 1'de görülen hedef elementlere ait etkin yük dağılımlarının gelen protonların enerjisinin artmasıyla birlikte artması hedeflerin özuyumlu potansiyel enerjileri ve iyonizasyon tesir kesitinin etkisi sonucu meydana gelmektedir. Ayrıca etkin yükün 1-10 MeV enerji aralığında değişmesi protonlarla hedef arasinda nükleer etkileşmelerden dolayı oluşacak radyoaktif ürünlerin meydana gelmesiyle açıklanabilir.

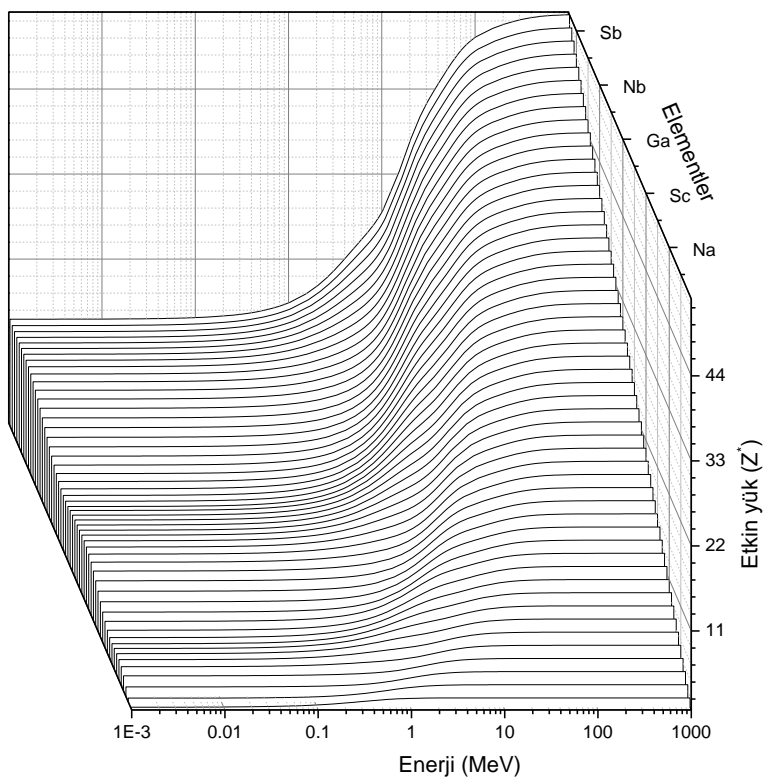

Şekil 1. Z=2-54 elementler için etkin yükün enerjiye göre değişimi. 


\subsection{Durdurma gücü}

Etkin yük yaklaşımı kullanılarak hesaplanan hedef atomların çarpışma durdurma gücü eğrileri Şekil 2 (a)'da görülmektedir. Şekilden durdurma gücü maksimum değerlerinin artan atom sayısı ile giderek azaldığı, maksimuma karşılık gelen enerji değerlerinin giderek arttığ1 açıkça anlaşılmaktadır. Bununla birlikte, düşük enerjilerde durdurma gücü eğrileri hızlı bir şekilde sıfıra gitmektedir. Helyum, neon gibi elementler normal koşullarda gaz halinde bulunmakla birlikte bu çalışmada katı bir hedef olarak dikkate alındı. Aslında gazlı bir ortamın durdurma gücü değerleri daha düşük çıkmasına rağmen bu çalışmadaki bütün hedef elementlerin katı halde olduğu varsayımı yapılarak helyum gibi elementlerin durdurma gücü değerleri diğer hedef elementlere göre daha yüksek çıkmıştır. Ayrıca bu çalışmada 40 keV'den düşük enerjilerde çarpışma durdurma gücü değerleri negatif değerler almaktadır.

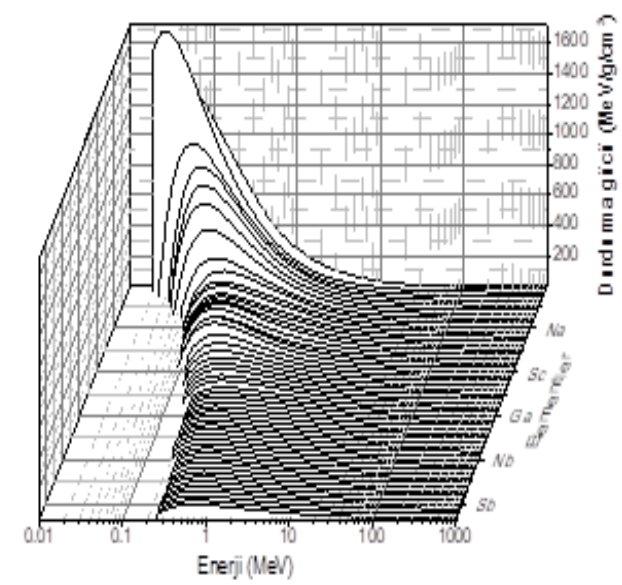

Şekil 2 (a). $1 \mathrm{keV}-1 \mathrm{GeV}$ enerjili protonlar için $Z=2-54$ elementlerin çarpışma durdurma gücü grafiği.

Şekil 2 (b)'de ise yapılan hesaplamaları kontrol etmek amaciyla karbon atomu için elde edilen karşılaştırmalı durdurma gücü değerleri görülmektedir. Sonuçlar literatürde mevcut SRIM(J.F. Ziegler et al., 2013), CasP (Grande \& Schiwietz, 2009), Janni (Janni, 1982) ve ICRU 49 (ICRU, 1993) verileriyle karşılaştırılmıştır.

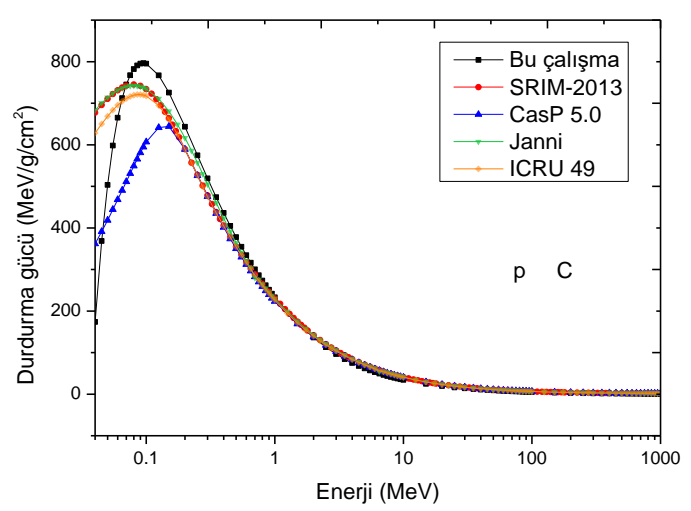

Şekil 2 (b). Karbon elementi için çarpışma durdurma gücünün karşılaştırılması.

Buna göre, karbon atomu için hesaplanan çarpışma durdurma gücü değerlerinin ICRU verileriyle olan uyum oranları 40-100 keV için 17.46, $100 \mathrm{keV}-1 \mathrm{MeV}$ için 6.87, 1-10 $\mathrm{MeV}$ için 9.15, $10 \mathrm{MeV}$ ve üzeri enerjiler için 18.49 olarak bulundu.

\section{Sonuç}

$\mathrm{Bu}$ çalışmada protonlar için periyodik cetvelde hidrojen hariç $Z=54$ 'e kadarki elementler için durdurma gücü hesaplamaları yapıldı. Hesaplamalar için Bethe-Bloch teorisi çatısı altında etkin yük yaklaşımı yöntemi kullanıld1. Buna göre gelen protonların enerjisiyle hedef elementlerin etkin yük ve durdurma gücü değerlerinin değişimi incelendi. Atom numarası ve gelen protonların enerjisi arttıkça etkin yükün arttığ1 görüldü. Durdurma gücü değerlerinin ise atom sayısı arttıkça azaldığı ve durdurma gücüne ait maksimum noktalarının yüksek enerjilere doğru kaydığı belirlendi. Elde edilen çarpışma durdurma gücü verilerinin doğruluğunu 
belirlemek amaciyla karbon atomu için hesaplanan değerler literatürde mevcut veri setleriyle karşılaştırıldı. Sonuçların ICRU değerleriyle hata oranlarının $100 \mathrm{keV}-10$ $\mathrm{MeV}$ enerji aralığı için \% 10'dan küçük, bu enerji aralığından küçük ve büyük değerlerde ise \% 10'dan büyük oldukları bulundu. Ayrıca yapılan çalışma, protonlar için etkin yük yaklaşımının $1 \mathrm{MeV}$ 'den düşük enerjilerde de belirli hata oranlarında kullanılabileceğini göstermiştir. Çalışmada bulunan hata oranlarının kaynağı olarak, hedef elementlerin etkin yük hesaplamaları için gerekli olan sıyrılma uzaklıklarının Newton-Raphson kök bulma yöntemi ile toplam enerji korunumu denklemi çözülerek bulunması sayılabilir. Bu değerler normalde Poisson denklemi çözülerek elde edilir. Fakat bu denklemin çözümü atom sayısının artmasıyla giderek zorlaşmaktadır.

Çarpışma durdurma gücünde elektronik yük yoğunluğu hesaplamaları için RHF yönteminde Slater tip orbitaller kullanıldı. $\mathrm{Bu}$ temel setler literatürde ksenon elementine kadar mevcuttur. Hesaplamaların periyodik cetveldeki çoğu elementlerle yapmak için Slater set takımları yerine Gaussian veya Slater-Gaussian orbitallerinin karışımı olan veri setlerinin sadece uzaysal kısmı değil küresel harmonikleri de dikkate alınarak kullanılması ve Poisson denkleminin çözülmesi için alternatif yöntemlerin tercih edilmesi yapılan bu çalışmayı daha da geliştirecektir. Etkin yük yaklaşımı ile daha önce Bragg toplama kuralı kullanılarak çeşitli insan organ dokuları ile ilgili hesaplamalar yapılmış olup, bu çalışma daha geniş bir enerji ve hedef aralı̆̆ çalışmada elde edilen sonuçlar yüklü parçacıkların madde ile etkileşmesinde radyasyon olaylarının açıklanmasına 1 şı tutacak, yüklü parçacık radyoterapi ve diğer nükleer

fizik

çalışmalarında

kullanılabilecektir.

\section{Kaynaklar}

Agostinelli, S., Allison, J., Amako, K., Apostolakis, J., Araujo, H., Arce, P., . . . Zschiesche, D. (2003). Geant4-a simulation toolkit. Nuclear Instruments and Methods in Physics Research Section A: Accelerators, Spectrometers, Detectors and Associated Equipment, 506(3), 250-303. doi:http://dx.doi.org/10.1016/S01689002(03)01368-8

Bohr, N. (1940). Scattering and Stopping of Fission Fragments. Phys. Rev., 58(7), 654$655 . \quad$ Retrieved from http://link.aps.org/doi/10.1103/PhysRev.58.6 54

Bohr, N. (1941). Velocity-Range Relation for Fission Fragments. Phys. Rev., 59(3), 270$275 . \quad$ Retrieved from http://link.aps.org/doi/10.1103/PhysRev.59.2 70

Bunge, C. F., Barrientos, J. A., \& Bunge, A. V. (1993). Roothaan-Hartree-Fock GroundState Atomic Wave Functions: Slater-Type Orbital Expansions and Expectation Values for $\mathrm{Z}=2-54$. Atomic Data and Nuclear Data Tables, 53(1), 113-162. doi:http://dx.doi.org/10.1006/adnd.1993.1003

Cabrera-Trujillo, R., Cruz, S. A., Oddershede, J., \& Sabin, J. R. (1997). Bethe theory of stopping incorporating electronic excitations of partially stripped projectiles. Physical Review A, 55(4), 2864-2872. Retrieved from http://link.aps.org/doi/10.1103/PhysRevA.55. 2864

Damache, S., Ouichaoui, S., Belhout, A., Medouni, A., \& Toumert, I. (2004). Stopping of $236 \mathrm{keV}-3.019 \mathrm{MeV}$ protons in mylar and polypropylene films. Nuclear Instruments and Methods in Physics Research Section B: Beam Interactions with Materials and Atoms, 225(4), 449-463. 
doi:http://dx.doi.org/10.1016/j.nimb.2004.06. For elements $1 \leqslant Z \leqslant 92$. Atomic Data and 007

Nuclear Data Tables, 27(2-3), 147-339. doi:http://dx.doi.org/10.1016/0092-

Demir, B., Sarpün, İ. H., Kaplan, A., Çapalı, 640X(82)90004-3

V., Aydın, A., \& Tel, E. (2015). Double

Differential Cross Section and Stopping Özdoğan, H., Çapalı, V., \& Kaplan, A. (2015).

Power Calculations of Light Charged Particle Reaction Cross-Section, Stopping Power and

Emission for the Structural Fusion Materials Penetrating Distance Calculations for the 50,52Cr. Journal of Fusion Energy, 34(4), Structural Fusion Material 54Fe in Different 808-816. doi:10.1007/s10894-015-9889-4

Reactions. Journal of Fusion Energy, 34(2), 379-385. doi:10.1007/s10894-014-9809-z

Dib, A., Ammi, H., Hedibel, M., Guesmia, A.,

Mammeri, S., Msimanga, M., \& Pineda- Pierce, T. E., Bowman, W. W., \& Blann, M. Vargas, C. A. (2015). Electronic stopping (1968). Stopping Powers of S32, C135, Br79, power data of heavy ions in polymeric foils in and I127 Ions in Mylar. Physical Review, the ion energy domain of LSS theory. Nuclear 172(2), 287-290. Retrieved from Instruments and Methods in Physics Research http://link.aps.org/doi/10.1103/PhysRev.172.2 Section B: Beam Interactions with Materials 87

and Atoms, 362, 172-181. doi:https://doi.org/10.1016/j.nimb.2015.09.083 Porter, L. E. (1980). Mean excitation energy of polystyrene extracted from proton-stoppingTransport Code : (program Version 2005): 22(5), 2221-2225. $\begin{gathered}\text { Retrieved from } \\ \text { Red }\end{gathered}$ CERN. http://link.aps.org/doi/10.1103/PhysRevB.22.2 221

Garcia-Molina, R., Abril, I., de Vera, P., \& Paul, H. (2013). Comments on recent Reynolds, H. K., Dunbar, D. N. F., Wenzel, W. measurements of the stopping power of liquid A., \& Whaling, W. (1953). The Stopping Cross water. Nuclear Instruments and Methods in Section of Gases for Protons, 30-600 kev. Physics Research Section B: Beam Interactions Physical Review, 92(3), 742-748. Retrieved with Materials and Atoms, 299(0), 51-53. from doi:http://dx.doi.org/10.1016/j.nimb.2013.01.0 http://link.aps.org/doi/10.1103/PhysRev.92.74 38 2

Grande, P. L., \& Schiwietz, G. (2009). Sarpün, İ. H., Aydın, A., Kaplan, A., Demir, B., Convolution approximation for the energy loss, Tel, E., \& Çapal1, V. (2015). Double ionization probability and straggling of fast Differential Charged Particle Emission Cross ions. Nuclear Instruments and Methods in Sections and Stopping Power Calculations for Physics Research Section B: Beam Interactions Structural Fusion Materials 58,60Ni. Journal of with Materials and Atoms, 267(6), 859-865. Fusion Energy, 34(6), 1306-1313. doi:http://dx.doi.org/10.1016/j.nimb.2009.02.0 doi:10.1007/s10894-015-9961-0 17

Sugiyama, H. (1981). Electronic stopping ICRU. (1993). Stopping Powers and Ranges for power formula for intermediate energies. Protons and Alpha Particles. Retrieved from Radiation Effects, 56(3-4), 205-211. doi:10.1080/00337578108229892

Janni, J. F. (1982). Energy loss, range, path length, time-of-flight, straggling, multiple Usta, M., \& Tufan, M. Ç. (2017). Stopping scattering, and nuclear interaction probability: power and range calculations in human tissues In two parts. Part 1. For 63 compounds Part 2. by using the Hartree-Fock-Roothaan wave 
functions. Radiation Physics and Chemistry, 140(Supplement $\quad$ C), 43-50. doi:https://doi.org/10.1016/j.radphyschem.201 7.03.005

Usta, M., Tufan, M. Ç., Aydın, G., \& Bozkurt, A. (2018). Stopping power and dose calculations with analytical and Monte Carlo methods for protons and prompt gamma range verification. Nuclear Instruments and Methods in Physics Research Section A: Accelerators, Spectrometers, Detectors and Associated Equipment, $\quad 897, \quad 106-113$. doi:https://doi.org/10.1016/j.nima.2018.04.045

Ziegler, J. F., Biersack, J. P., \& Ziegler, M. D. (2013). SRIM, the Stopping and Range of Ions in Matter: SRIM Company.

Ziegler, J. F., \& Manoyan, J. M. (1988). The stopping of ions in compounds. Nuclear Instruments and Methods in Physics Research Section B: Beam Interactions with Materials and Atoms, 35(3-4), 215-228. doi:http://dx.doi.org/10.1016/0168583X(88)90273-X 